TRANSACTIONS OF THE

AMERICAN MATHEMATICAL SOCIETY

Volume 362, Number 12, December 2010, Pages 6261-6277

S 0002-9947(2010)04897-4

Article electronically published on July 14, 2010

\title{
RELATIONSHIP BETWEEN SCATTERING MATRIX AND SPECTRUM OF QUANTUM GRAPHS
}

\author{
G. BERKOLAIKO AND B. WINN
}

\begin{abstract}
We investigate the equivalence between spectral characteristics of the Laplace operator on a metric graph and the associated unitary scattering operator. We prove that the statistics of level spacings and moments of observations in the eigenbases coincide in the limit that all bond lengths approach a positive constant value.
\end{abstract}

\section{INTRODUCTION}

Quantum graphs have attracted much attention in recent years due both to their applicability as physical models and their interesting mathematical properties. We refer the reader to the edited volumes [3, 11] for a potpourri of new results. Recent reviews, dedicated to quantum graphs, include [25, 17].

In this article we focus on one feature of interest which is the use of graph models to probe the universality of quantum systems. One of the unsolved paradoxes of quantum mechanics is the observation that a great many quantum systems are remarkably similar when one makes statistical observations in the semi-classical régime. This manifests itself both in the energy levels and associated energy eigenfunctions. Despite a great deal of effort, this universality is poorly understood mathematically. Generic quantum graphs exhibit this universal behavior and represent the most likely system for which a full mathematically rigorous proof of this universality will first be found. Important steps in this direction have been taken in [16].

A quantum graph can be defined in two different, but related, ways (a complete description appears in the following section). One may consider either a self-adjoint realisation of the Laplace operator or a scattering matrix approach. Mathematically, the scattering approach appears to be more tractable and has formed the basis of most investigations [37, 40, 7, 2, 5]. Moreover, the spectra arising from these quantizations are subtly different. This is not as confusing as it might seem, since the statistical properties of both versions of the spectrum are believed to coincide when averaged over a large interval. It is the purpose of this article to put a concrete mathematical foundation behind this belief.

The plan of the article is as follows: In the next section we give precise definitions of the two ways to describe quantum graphs, and in Section 3 describe our main results. In Section 4 we describe the tools used and then present in Section 5 the proofs of our results.

Received by the editors March 21, 2008.

2000 Mathematics Subject Classification. Primary 34L20, 81Q10, 81Q50.

(C)2010 American Mathematical Society Reverts to public domain 28 years from publication 


\section{TWO DESCRIPTIONS OF QUANTUM GRAPHS}

For both constructions of a quantum graph we begin with a graph $G=(\mathcal{V}, \mathcal{B})$ where $\mathcal{V}$ is a finite set of vertices (sometimes referred to as nodes) and $\mathcal{B}$ is the set of bonds (or edges). Each bond $b$ has a positive length, denoted $L_{b}$. The total number of bonds is $B$. We denote by $d_{v}$ the degree of the vertex $v \in \mathcal{V}$, which is the number of bonds emanating from it.

2.1. The Laplace operator approach. The first way to define a quantum graph is to identify each bond $b$ with the interval $\left[0, L_{b}\right]$ of the real line and thus define the $L^{2}$-space of functions on the graph. Then one can consider the eigenproblem

$$
-\frac{\mathrm{d}^{2}}{\mathrm{~d} x^{2}} u_{b}(x)=\lambda^{2} u_{b}(x) .
$$

This setup has a long history of being used in physical models [33, 19, 36]. It was studied by mathematicians since at least the 1980s [29, 35, 41, 32, 34, 15, 12]. The study of spectral statistics of quantum graphs was initiated in [23, 24].

To make the operator in (2.1) self-adjoint one needs to impose matching conditions on the behavior of $u$ at the vertices of the graph. One possibility is to impose Kirchhoff conditions 1 We require that $u$ is continuous at the vertices and that the probability current is conserved, i.e.

$$
\sum_{v \in b} \frac{\mathrm{d}}{\mathrm{d} x} u_{b}(v)=0 \quad \text { for all } v \in \mathcal{V},
$$

where the sum is over all bonds that originate from the vertex $v$ and the derivatives are taken at the vertex $v$ in the outward direction. The admissible matching conditions were classified in, among other sources, [21, 20, 26.

A solution to the eigenvalue equation (2.2) on the bond $b$ can be written as a linear combination of plane waves,

$$
u_{b}\left(x_{b}\right)=c_{b} \mathrm{e}^{\mathrm{i} \lambda x_{b}}+\hat{c}_{b} \mathrm{e}^{-\mathrm{i} \lambda x_{b}} .
$$

A solution on the whole graph can be uniquely defined by specifying the corresponding vector of coefficients $\mathbf{c}=\left(c_{1}, \ldots, c_{B}, \hat{c}_{1}, \ldots, \hat{c}_{B}\right)^{T}$. The elements of the vector c are naturally associated with directed bonds of the graph $G$.

Imposing the matching conditions, we find that $\lambda^{2}$ is an eigenvalue if and only if $S(\lambda) \mathbf{c}=\mathbf{c}$ for some explicitly given matrix $S(\lambda)$. For instance, in the case of Kirchhoff conditions given above,

$$
S(\lambda)=\mathrm{e}^{\mathrm{i} \lambda \mathbf{L}} S_{0},
$$

where the elements of the $2 B \times 2 B$ matrix $S_{0}$ are given by

$$
\left(S_{0}\right)_{\left(v_{1}, v_{2}\right)\left(v_{3}, v_{4}\right)}=\delta_{v_{2}, v_{3}}\left(\frac{2}{d_{v_{2}}}-\delta_{v_{1}, v_{4}}\right),
$$

for $\left(v_{1}, v_{2}\right)$ and $\left(v_{3}, v_{4}\right)$ directed bonds of the graph. By $\mathbf{L}$ we denoted the vector of bond lengths $\mathbf{L}=\left(L_{1}, \ldots, L_{B}\right)$ and defined, in a slight abuse of notation, a $2 B \times 2 B$

\footnotetext{
${ }^{1}$ Sometimes called "Neumann" conditions.
} 
diagonal matrix

$$
\mathrm{e}^{\mathrm{ix}}:=\left(\begin{array}{cccccc}
\mathrm{e}^{\mathrm{i} x_{1}} & & & & & \\
& \ddots & & & & \\
& & \mathrm{e}^{\mathrm{i} x_{B}} & & & \\
& & & \mathrm{e}^{\mathrm{i} x_{1}} & & \\
& & & & \ddots & \\
& & & & & \mathrm{e}^{\mathrm{i} x_{B}}
\end{array}\right),
$$

where $\mathbf{x} \in \mathbb{R}^{B}$. Note the doubling of dimension which signifies the move from undirected to directed bonds.

The general conditions for factorization (2.4) are given in 22, 14. Even when these conditions are not satisfied and $S$ depends on $\lambda$ in a non-trivial fashion, the above factorization is valid in the limit $\lambda \rightarrow \infty$. Thus, for the purpose of studying the spectral statistics it is not a strong restriction to assume that (2.4) applies, as we will do below.

2.2. The scattering approach. The second construction considers wave propagation on the graph where each vertex is treated as a scatterer and propagation along the bonds is free. This construction was first considered in the context of studying spectral statistics in 24] and then generalized in [39] to directed graphs. On each bond the waves travel in both directions. Therefore, at any given moment, the system is fully specified by a $2 B$-dimensional vector of wave amplitudes $\mathbf{a}$, indexed again by the directed bonds. Scattering at vertices is described by a unitary matrix $T$, having the property that

$$
T_{\left(v_{1}, v_{2}\right)\left(v_{3}, v_{4}\right)}=0 \quad \text { if } \quad v_{2} \neq v_{3} .
$$

The free propagation results in the amplitude $a_{b}$ (here $b$ is a directed bond) being multiplied by the phase factor $\mathrm{e}^{\mathrm{i} \lambda L_{b}}$. Altogether we arrive at the quantum evolution operator $U(\lambda)=\mathrm{e}^{\mathrm{i} \lambda \mathbf{L}} T$.

Thus, in both constructions one ends up with a unitary matrix $S(\lambda)=\mathrm{e}^{\mathrm{i} \lambda \mathbf{L}} S_{0}$. This matrix specifies the eigenvalues $\left\{\lambda_{n}\right\}$ via the equation

$$
\operatorname{det}\left[I-\mathrm{e}^{\mathrm{i} \lambda \mathbf{L}} S_{0}\right]=0 .
$$

Actually, it was noted in [28] (see also [17, 14]) that the multiplicity of $\lambda=0$ as a root of (2.6) can be different from its multiplicity as an eigenvalue of the Laplace operator, but that for all positive eigenvalues the multiplicities coincide. To avoid this ambiguity we will omit the zero eigenvalues from the spectra that we consider; i.e. in our counting, $\lambda_{1}$ will be the first strictly positive eigenvalue. Since the multiplicity of the zero eigenvalue is necessarily finite (see Corollary 4.2 below), this omission will not affect any spectral statistics.

2.3. Spectral quantities for quantum graphs. In various sources the notion of the "spectrum $\sigma(G)$ of the graph $G$ " can refer to:

(1) the eigenproblem (2.1) and thus solutions $\left\{\lambda_{n}\right\}$ of (2.6),

(2) the eigenphases of the matrix $S(\lambda)$ for an arbitrary $\lambda$, i.e. to the set of $2 B$ numbers $\left\{\theta_{j}\right\}$ such that $\mathrm{e}^{\mathrm{i} \theta_{j}}$ is the $j$-th eigenvalue of $S(\lambda)$.

To distinguish the two notions of the spectrum we will refer to the first definition above as the $\lambda$-spectrum and to the second as the $\theta$-spectrum. 
In a similar way, the "eigenvector" of $G$ can refer to one of three objects:

(1) the function $u(x)$ that solves (2.1), subject to boundary conditions, for some $\lambda_{n}$ in the $\lambda$-spectrum,

(2) the eigenvector of $S\left(\lambda_{n}\right)$ corresponding to the eigenvalue 1, i.e. to the solution $\mathbf{c}$ of $S\left(\lambda_{n}\right) \mathbf{c}=\mathbf{c}$. These eigenvectors will be denoted by $\phi_{n}$,

(3) any of the $2 B$ eigenvectors of $S(\lambda)$ for arbitrary $\lambda$, denoted by $\boldsymbol{\psi}_{j}(\lambda)$, $j=1, \ldots, 2 B$.

The correspondence between the first two types of eigenvectors is given by formula (2.3). A heuristic formula which connects properties of the second and the third types of eigenvectors was suggested in [5, Eq. (5)]. In this article we give a rigorous derivation of the formula.

\section{MAin Results}

Our results concern equivalences between statistical properties of two types of eigenvalues and eigenvectors of quantum graphs. These results are derived as an application of Proposition 4.4 introduced in Section 4

3.1. Eigenvalue statistics. Starting with the seminal work of Kottos and Smilansky [23, 24], quantum graphs became a popular model of quantum chaos. One of the more pertinent questions of quantum chaos is the universality of the eigenvalue correlations among systems of a certain type. On graphs, 23. showed some preliminary numerical evidence that the eigenvalue spectrum of graphs follows the general prediction [8, 9] which says that the spectrum of a classically chaotic system should have correlations typically associated to eigenvalues of large random matrices. Persistent deviations from the predicted behavior were found in star graphs [24, 4, and Tanner [40] proposed a precise condition on the graphs to follow the random matrix theory prediction. This question was then attacked analytically by various methods, with results reported, in particular, in [7, 16, 2. For more information we refer the reader to a recent review [17.

While the original interest was in level statistics, i.e. the statistical functions of the spectrum $\left\{\lambda_{n}\right\}$, most of the analytical studies were in fact concentrating on the eigenphase statistics of the $\theta$-spectrum. There are heuristic reasons why the corresponding statistics should coincide, but the formal link between the two has hitherto not been explored.

In this article we prove that, in the correct limit, the two statistics are equivalent. We take the example of nearest-neighbor spacing distribution, i.e. the distribution of gaps $\left\{\lambda_{n}-\lambda_{n-1}\right\}$ on one hand and the values of the functions $\left\{\theta_{j}(\lambda)-\theta_{j-1}(\lambda)\right\}$ on the other. Formally, the level spacing distribution is given by

$$
P(s)=\lim _{N \rightarrow \infty} \frac{1}{N} \sum_{n=1}^{N} \delta\left(s-\left(\lambda_{n}-\lambda_{n-1}\right)\right),
$$

where $\delta$ is the Dirac delta-function. Mathematically, the distributions are defined via a family of test functions.

Theorem 3.1. Let $\mathbf{L}$ be linearly independent over $\mathbb{Q}$ and $\bar{L}=\left(L_{1}+\ldots+L_{B}\right) / B$ denote the mean bond length. If $h$ is a continuous function, then the limits

$$
P_{\lambda}[h]:=\lim _{N \rightarrow \infty} \frac{1}{N} \sum_{n=1}^{N} h\left(\bar{L}\left(\lambda_{n}-\lambda_{n-1}\right)\right)
$$


and

$$
P_{\theta}[h]=\lim _{\Lambda \rightarrow \infty} \frac{1}{\Lambda} \int_{0}^{\Lambda} \frac{1}{2 B} \sum_{j=1}^{2 B} h\left(\theta_{j}(\lambda)-\theta_{j-1}(\lambda)\right) \mathrm{d} \lambda
$$

exist and define bounded linear functionals of $h$. If, for some $\ell_{0}>0$, we take the limit $\mathbf{L} \rightarrow \ell_{0}(1,1, \ldots, 1)$ while keeping $\mathbf{L}$ linearly independent over $\mathbb{Q}$, the two functionals coincide. Namely,

$$
\lim _{\Delta L \rightarrow 0} P_{\lambda}[h]=\lim _{\Delta L \rightarrow 0} P_{\theta}[h],
$$

where $\Delta L:=L_{\max }-L_{\min }$ is the spread of the distribution of the bond lengths.

Remark 3.2. If $\mathbf{L}$ is not linearly independent over $\mathbb{Q}$, the limits (3.1) and (3.2) still exist but are highly sensitive to the changes in individual bond length. Thus the limits in equation (3.3) are, in general, singular. In particular, when all bond lengths are equal $(\Delta L=0)$, the $\lambda$-spectrum is periodic and spacings in the $\theta$ spectrum are independent of $\lambda$. The nearest-neighbor distribution in this case is highly degenerate.

Remark 3.3. It is straightforward to extend the result to the $r$-th nearest-neighbor spacing distributions, i.e. the distributions of $\lambda_{n}-\lambda_{n-r}$ and $\theta_{j}(\lambda)-\theta_{j-r}(\lambda)$. Moreover, when all the $r$-th nearest-neighbor spacing distributions coincide, so do other statistical functions such as the $n$-point correlation functions. (See, for example, equation (6.1.39) of [30.)

3.2. Eigenvector statistics. The equivalence between the statistics of the $\lambda$ spectrum and the $\theta$-spectrum can be extended to eigenfunction statistics.

To proceed, we need to introduce some notation in addition to that introduced previously in Section 2.3. By $\boldsymbol{\psi}_{j}(\lambda)$ we will denote the $j$-th eigenvector of $S(\lambda)$. As before, $\mathbf{L}$ denotes the vector of the bond lengths. The total length of the graph is $\mathscr{L}=\sum_{b=1}^{B} L_{b}$, and the average bond length, $\mathscr{L} / B$, is denoted by $\bar{L}$.

Let $A$ be a $2 B \times 2 B$ matrix ("observable"). We denote $A_{n}=\left\langle\phi_{n}|A| \phi_{n}\right\rangle$ and $A_{j}(\lambda)=\left\langle\boldsymbol{\psi}_{j}(\lambda)|A| \boldsymbol{\psi}_{j}(\lambda)\right\rangle$, which corresponds to the "expected value of the observable $A$ ". We define the bond length observable by $L:=\operatorname{diag}(\mathbf{L}, \mathbf{L})$ and set $L\left(\lambda_{n}\right)=\left\langle\phi_{n}|L| \phi_{n}\right\rangle$.

Further, let $D$ denote the $2 B \times 2 B$ random diagonal unitary matrix

$$
D:=\mathrm{e}^{\mathrm{i} \mathbf{X}},
$$

where $\mathbf{X}$ is a random vector distributed uniformly on $\mathbb{T}^{B}:=[0,2 \pi)^{B}$, and let $A_{j}(D)=$ $\left\langle\boldsymbol{\psi}_{j}(D)|A| \boldsymbol{\psi}_{j}(D)\right\rangle$ be the expectation of $A$ with respect to the $j$-th eigenvalue of the matrix $D S_{0}$. By $\mathbb{E}^{D}(\cdot)$ we denote averages with respect to the distribution of $D$.

One of the central results in the study of statistical properties of eigenvectors is "quantum ergodicity". Quantum ergodicity is the property of almost all eigenvectors to equidistribute [38, 43, 10]. In our context, this corresponds to the vanishing of the variance of either $A_{n}$ or $A_{j}(\lambda)$ in some limit. Here we shall not discuss the important question of which limit is appropriate; we refer the interested reader to [5]. Instead we prove that the variances (as indeed all other moments) of $A_{n}$ and $A_{j}(\lambda)$ are equivalent. 
Theorem 3.4. Let $\mathbf{L}$ be linearly independent over $\mathbb{Q}$. Then, for all $m \geq 0$,

$\lim _{N \rightarrow \infty} \frac{1}{N} \sum_{n=1}^{N} \frac{A_{n}^{m}}{L\left(\lambda_{n}\right) / \bar{L}}=\lim _{\Lambda \rightarrow \infty} \frac{1}{\Lambda} \int_{0}^{\Lambda} \frac{1}{2 B} \sum_{j=1}^{2 B} A_{j}(\lambda)^{m} \mathrm{~d} \lambda=\mathbb{E}^{D}\left(\frac{1}{2 B} \sum_{j=1}^{2 B} A_{j}(D)^{m}\right)$.

The weighting on the left-hand side of (3.4) shows that there is an exact equivalence of moments only in the (additional) limit $\Delta L \rightarrow 0$, as was taken in [4, 6]. However, provided $\Delta L$ is bounded away from zero and infinity, the centered moments would approach the zero limit either simultaneously or not at all. Another important feature of equation (3.4) is that its second part relates the moments of the eigenvectors of a fixed graph to the averaged properties of an ensemble of random matrices. While such averaging was not necessary in the proof of quantum ergodicity of models considered in [5], it is expected to be helpful in more general circumstances.

\section{Main tools}

4.1. Graph spectra and torus flow. Our methods are based on an idea going back to Barra and Gaspard [1, who suggested viewing the eigenvalues $\lambda_{n}$ as the Poincaré return times of a flow to a hypersurface defined by an extension of equation (2.6).

Let $\mathbb{T}^{B}$ be the torus of side $2 \pi$ in $B$ dimensions. Define the surface $\Sigma \subseteq \mathbb{T}^{B}$ by

$$
\Sigma:=\left\{\mathbf{x}: \operatorname{det}\left[\mathrm{e}^{\mathrm{ix}} S_{0}-I\right]=0\right\},
$$

where we use notation (2.5) for the exponential of a vector.

Further, define a flow $\phi_{t}\left(\mathbf{x}_{0}\right)$ on $\mathbb{T}^{B}$ by

$$
\phi_{t}\left(\mathbf{x}_{0}\right)=\mathbf{x}_{0}+t \mathbf{L} \bmod 2 \pi \text {. }
$$

If the components of $\mathbf{L}$ are linearly independent over $\mathbb{Q}$, then the flow is equidistributing on the torus.

Since $\phi_{\lambda}(\mathbf{0})=\lambda \mathbf{L} \bmod 2 \pi$, equation (2.6) can be rewritten as

$$
\operatorname{det}\left[\mathrm{e}^{\mathrm{i} \phi_{\lambda}(\mathbf{0})} S_{0}-I\right]=0,
$$

or simply as $\phi_{\lambda}(\mathbf{0}) \in \Sigma$. Thus the times $t=\lambda_{n}, n \in \mathbb{Z}$, of intersections of the flow $\phi_{t}(\mathbf{0})$ with the surface $\Sigma$ give the set of points in the quantum spectrum of a graph (see Figure 1).

The idea of Barra and Gaspard [1] was to use the ergodicity of the flow (4.2) to compute averages like (3.1) as an integral over the surface $\Sigma$. In this paper we put their idea on a mathematical footing (in particular, tackling the problem of using the ergodic theorem to integrate over $\Sigma$ which is measure zero subset of $\mathbb{T}^{B}$ ) and extend it to apply to the problems described in Section 3 .

4.2. More about intersections with $\Sigma$. The following result goes back to at least [24, Eq. (70)]. For completeness, we provide it with a proof.

Lemma 4.1. Let $U$ be $a 2 B \times 2 B$ unitary matrix, and let $D(t):=\mathrm{e}^{\mathrm{i} \phi_{t}\left(\mathrm{x}_{0}\right)}$ for fixed $\mathbf{x}_{0}$. Let $\mathrm{e}^{\mathrm{i} \theta(t)}$ be an eigenvalue of the unitary matrix $D(t) U$ and $\mathbf{u}(t)$ be the corresponding normalised eigenvector. Then,

$$
\frac{\mathrm{d} \theta}{\mathrm{d} t}=\langle\mathbf{u}(t)|L| \mathbf{u}(t)\rangle,
$$



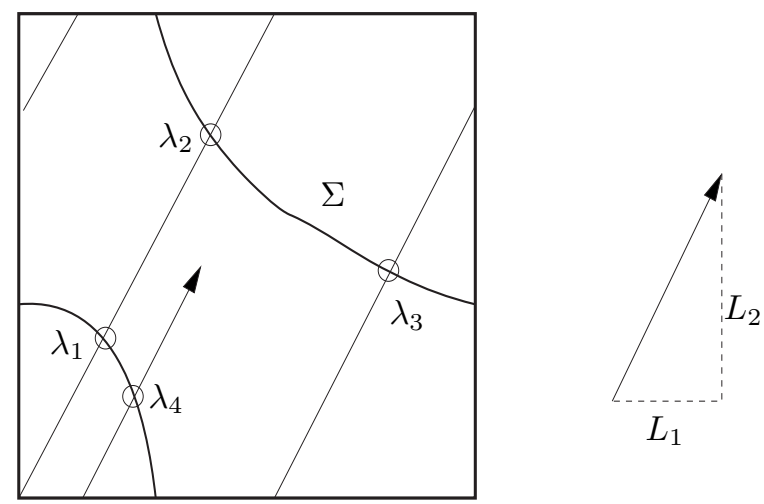

Figure 1. A cartoon of the flow piercing the surface $\Sigma$. The direction of increasing $k$ is indicated by an arrow, and eigenvalues are indicated by little circles.

where $L=\operatorname{diag}(\mathbf{L}, \mathbf{L})$. In particular,

$$
L_{\min } \leq \frac{\mathrm{d} \theta}{\mathrm{d} t} \leq L_{\max }
$$

Proof. Since $D(t) U$ is unitary and analytic, the function $\theta(t)$ can be chosen real analytic, and so there are no problems differentiating the eigenvalue equation,

$$
D(t) U \mathbf{u}(t)=\mathrm{e}^{\mathrm{i} \theta(t)} \mathbf{u}(t),
$$

with respect to $t$ to obtain

$$
\mathrm{i} L D(t) U \mathbf{u}(t)+D(t) U \mathbf{u}^{\prime}(t)=\mathrm{i} \theta^{\prime}(t) \mathrm{e}^{\mathrm{i} \theta(t)} \mathbf{u}(t)+\mathrm{e}^{\mathrm{i} \theta(t)} \mathbf{u}^{\prime}(t) .
$$

Multiplying by $\mathbf{u}(t)^{\dagger}$ (the conjugate transpose of $\mathbf{u}(t)$ ),

$$
\mathrm{ie}^{\mathrm{i} \theta(t)} \mathbf{u}(t)^{\dagger} L \mathbf{u}(t)+\mathbf{u}(t)^{\dagger} D(t) U \mathbf{u}^{\prime}(t)=\mathrm{i} \theta^{\prime}(t) \mathrm{e}^{\mathrm{i} \theta(t)},
$$

where we used that $\mathbf{u}(t)^{\dagger} \mathbf{u}(t)=1$ and $\mathbf{u}(t)^{\dagger} \mathbf{u}^{\prime}(t)=0$. To handle the second term on the left-hand side we notice that

$$
\mathbf{u}(t)^{\dagger} D(t) U=\left((D(t) U)^{\dagger} \mathbf{u}(t)\right)^{\dagger}=\left(\mathrm{e}^{-\mathrm{i} \theta(t)} \mathbf{u}(t)\right)^{\dagger}=\mathrm{e}^{\mathrm{i} \theta(t)} \mathbf{u}(t)^{\dagger},
$$

and so

$$
\mathbf{u}(t)^{\dagger} D(t) U \mathbf{u}^{\prime}(t)=\mathrm{e}^{\mathrm{i} \theta(t)} \mathbf{u}(t)^{\dagger} \mathbf{u}^{\prime}(t)=0 .
$$

Thus,

$$
\frac{\mathrm{d} \theta}{\mathrm{d} t}=\mathbf{u}(t)^{\dagger} L \mathbf{u}(t)=\sum_{j=1}^{B} L_{j}\left(\left|u(t)_{j}\right|^{2}+\left|u(t)_{j+B}\right|^{2}\right),
$$

leading to the desired bounds.

We can fix bounds on the rate of crossing of the flow with $\Sigma$ based on the fact that

$$
\operatorname{det}\left[\mathrm{e}^{\mathrm{i} \phi_{t}\left(\mathbf{x}_{0}\right)} S_{0}-I\right]=0
$$

if and only if one of the eigenphases $\theta(t)$ is equal to a multiple of $2 \pi$. Since all $\theta(t)$ are increasing functions and Lemma 4.1 gives us an estimate of the increase, we get the following corollary. 
Corollary 4.2. If $t$ varies in an interval of length $\frac{2 \pi}{L_{\min }}$, then the flow $\phi_{t}\left(\mathbf{x}_{0}\right)$ intersects the surface $\Sigma$ at least $2 B$ times.

If $t$ varies in an interval of length $\frac{2 \pi}{L_{\max }}$, then the flow $\phi_{t}\left(\mathbf{x}_{0}\right)$ intersects the surface $\Sigma$ at most $2 B$ times.

On average, the flow $\phi_{t}\left(\mathbf{x}_{0}\right)$ intersects the surface $2 B$ times in an interval of length $\frac{2 \pi}{\bar{L}}$, where $\bar{L}$ is the average bond length. This is a consequence of the asymptotic density of the $\lambda$-spectrum, the Weyl law for graphs. This classical result has been known for graphs for some time. A particularly elegant proof has appeared recently in [18, Theorem 1].

Proposition 4.3. Let $N(\Lambda):=\#\left\{0<\lambda_{n} \leq \Lambda\right\}$, where spectral points are counted with multiplicity. Then

$$
N(\Lambda) \sim \frac{\mathscr{L}}{\pi} \Lambda \quad \text { as } \Lambda \rightarrow \infty,
$$

where $\mathscr{L}:=L_{1}+\cdots+L_{B}$.

We remark that, in fact, the stronger asymptotic

$$
N(\Lambda)=\frac{\mathscr{L}}{\pi} \Lambda+\mathrm{O}(1)
$$

is proved in [18; however, we shall only make use of (4.10).

4.3. The main proposition. Let $\Phi$ be a bounded function, defined on $\Sigma$. We extend it to the whole of $\mathbb{T}^{B}$, by the following "thickening". Let $\epsilon>0$ and define

$$
\Phi_{\epsilon}(\mathbf{x}):=\sum_{\boldsymbol{\xi} \in \Sigma} \Phi(\boldsymbol{\xi}) \mathbb{I}_{\{\boldsymbol{\xi}=\mathbf{x}+t \mathbf{L}:|t| \leq \epsilon / 2\}} .
$$

Thus $\Phi_{\epsilon}$ takes the value $\Phi(\boldsymbol{\xi})$ at all points within a distance at most $\epsilon / 2$ from $\boldsymbol{\xi} \in \Sigma$ in the direction of the flow. Should two or more different branches of the surface $\Sigma$ come close together, or intersect, the function $\Phi_{\epsilon}$ takes the sum of the values of $\Phi(\boldsymbol{\xi})$ on the branches (see Figure 2). The sum in (4.11) is finite as a consequence of Corollary 4.2 .

We permit the function $\Phi$ to be undefined on a set $\mathcal{U} \subseteq \Sigma$, provided that $\mathcal{U}$ is small in the following sense. The projection of the set $\mathcal{U}$ onto the $B$-1-dimensional hyperplane $P:=\left\{\mathbf{x} \in \mathbb{T}^{B}: x_{1}=0\right\}$ is required to have measure zero:

$$
\text { meas }\left\{\mathbf{y} \in P: \exists t, 0<t \leq 2 \pi / L_{1}, \phi_{t}(\mathbf{y}) \in \mathcal{U}\right\}=0 .
$$

This ensures that the discontinuities of $\Phi_{\epsilon}$ have measure zero with respect to $\Sigma$.

Our main tool is the following proposition.

Proposition 4.4. Let $\mathbf{L}$ be linearly independent over $\mathbb{Q}$ and let $\Phi$ be defined on all of $\Sigma$, except possibly for a set $\mathcal{U}$ satisfying (4.12), and be positive and bounded. Denote by $t_{n}=t_{n}\left(\mathbf{x}_{0}\right)$ the consecutive intersection times of the flow $\phi_{t}\left(\mathbf{x}_{0}\right)$ with the surface $\Sigma$. Then for any $\mathbf{x}_{0} \in \mathbb{T}^{B}$ and $\epsilon>0$

$$
\lim _{N \rightarrow \infty} \frac{1}{N} \sum_{n=1}^{N} \Phi\left(\phi_{t_{n}}\left(\mathbf{x}_{0}\right)\right)=\frac{1}{\bar{d}(2 \pi)^{B} \epsilon} \int_{\mathbb{T}^{B}} \Phi_{\epsilon}(\mathbf{x}) \mathrm{d} \mathbf{x},
$$

where $\bar{d}:=\lim _{n \rightarrow \infty} n / t_{n}=\mathscr{L} / \pi$ is the mean density of the intersections $t_{n}$. 


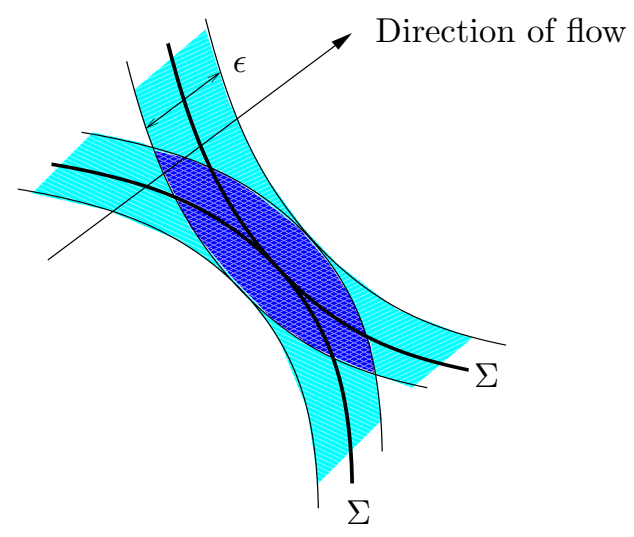

Figure 2. The region in which the "thickened" function $\Phi_{\epsilon}$ is supported. If two branches of $\Sigma$ come close together, the function takes the sums of the values on the different branches. In the figure this region is shaded in a darker tone.

Remark 4.5. Since we want the result for every point $\mathbf{x}_{0} \in \mathbb{T}^{B}$, simple ergodicity of the flow $\phi_{t}$ would not be enough. Instead we shall use Weyl's equidistribution which applies to intervals (and Riemann integrable functions) rather than Lebesgue measurable sets (and integrable function). Namely we use the following lemma, which follows directly from the results of Weyl [42].

Lemma 4.6. Let $G: \mathbb{T}^{B} \rightarrow \mathbb{R}$ be a Riemann integrable function. Then, for every $\mathbf{x}_{0} \in \mathbb{T}^{B}$,

$$
\lim _{T \rightarrow \infty} \frac{1}{T} \int_{0}^{T} G\left(\phi_{t}\left(\mathbf{x}_{0}\right)\right) \mathrm{d} t=\frac{1}{\left|\mathbb{T}^{B}\right|} \int_{\mathbb{T}^{B}} G(\mathbf{x}) \mathrm{d} \mathbf{x} .
$$

If the integral on the left-hand side does not exist, equation (4.14) should be understood in terms of upper and lower Darboux integrals (limits of Darboux sums),

$$
\lim _{T \rightarrow \infty} \frac{1}{T} \text { L.D. } \int_{0}^{T} G\left(\phi_{t}\left(\mathbf{x}_{0}\right)\right) \mathrm{d} t=\lim _{T \rightarrow \infty} \frac{1}{T} \text { U.D. } \int_{0}^{T} G\left(\phi_{t}\left(\mathbf{x}_{0}\right)\right) \mathrm{d} t=\frac{1}{\left|\mathbb{T}^{B}\right|} \int_{\mathbb{T}^{B}} G(\mathbf{x}) \mathrm{d} \mathbf{x} .
$$

Proof. The proof follows the standard procedure; see for example [27, Theorem 1.1]. Let

$$
\mathbb{T}^{B}=\bigcup_{j} R_{j}
$$

be a finite partition of $\mathbb{T}^{B}$ into well-behaved sets, such as rectangles. Then

$$
G(\mathbf{x}) \leq \hat{G}(\mathbf{x}):=\sum_{j} \mathbb{I}_{R_{j}}(\mathbf{x}) \sup _{\mathbf{y} \in R_{j}} G(\mathbf{y}),
$$

where $\mathbb{I}_{A}$ is the indicator function of set $A$. By [42, Satz 5],

$$
\lim _{T \rightarrow \infty} \frac{1}{T} \int_{0}^{T} \mathbb{I}_{R_{j}}\left(\phi_{t}\left(\mathbf{x}_{0}\right)\right) \mathrm{d} t=\frac{\left|R_{j}\right|}{\left|\mathbb{T}^{B}\right|}
$$


the volume of the set $R_{j}$. Therefore,

$$
\begin{aligned}
\limsup _{T \rightarrow \infty} \frac{1}{T} \mathrm{U} \cdot \mathrm{D} \cdot \int_{0}^{T} G\left(\phi_{t}\left(\mathbf{x}_{0}\right)\right) \mathrm{d} t \leq \lim _{T \rightarrow \infty} \frac{1}{T} \int_{0}^{T} \hat{G}\left(\phi_{t}\left(\mathbf{x}_{0}\right)\right) \mathrm{d} t \\
=\frac{1}{\left|\mathbb{T}^{B}\right|} \sum_{j}\left|R_{j}\right| \max _{\mathbf{y} \in R_{j}} G(\mathbf{y}),
\end{aligned}
$$

which is the upper Darboux sum for the integral $|\mathbb{T}|^{-B} \int_{\mathbb{T}^{B}} G(\mathbf{x}) \mathrm{d} \mathbf{x}$. Since $G$ is Riemann integrable, the Darboux sums converge to the value of the integral in the limit as the partition (4.16) becomes finer. Analogous lower estimates lead to (4.15).

Proof of Proposition 4.4. Since the function $\Phi_{\epsilon}$ is bounded and the discontinuity set has measure zero, $\Phi_{\epsilon}$ is Riemann integrable and we can apply Lemma 4.6. According to the way that $\Phi_{\epsilon}$ is a thickening of width $\epsilon$ of $\Phi$ on $\Sigma$, we have that

$$
\frac{1}{N} \sum_{n=1}^{N} \Phi\left(\phi_{t_{n}}\left(\mathbf{x}_{0}\right)\right)=\frac{1}{N \epsilon} \int_{0}^{t_{N}+\epsilon / 2} \Phi_{\epsilon}\left(\phi_{t}\left(\mathbf{x}_{0}\right)\right) \mathrm{d} t+\mathrm{O}\left(\frac{1}{N}\right)
$$

as $N \rightarrow \infty$. The error term comes from a possible overlap of the integration range with crossings other than $t_{n}, 1 \leq n \leq N$. Corollary 4.2 guarantees that the number of such crossings is uniformly bounded.

Now we let $N \rightarrow \infty$, which entails $T:=t_{N}+\epsilon / 2 \rightarrow \infty$, and apply Lemma 4.6.

$$
\begin{aligned}
\lim _{N \rightarrow \infty} \frac{1}{N} \sum_{n=1}^{N} \Phi\left(\phi_{\lambda_{n}}\left(\mathbf{x}_{0}\right)\right) & =\lim _{N \rightarrow \infty} \frac{t_{N}}{N \epsilon} \lim _{T \rightarrow \infty} \frac{1}{T} \int_{0}^{T} \Phi_{\epsilon}\left(\phi_{t}\left(\mathbf{x}_{0}\right)\right) \mathrm{d} t \\
& =\frac{1}{\bar{d}(2 \pi)^{B} \epsilon} \int_{\mathbb{T}^{B}} \Phi_{\epsilon}(\mathbf{x}) \mathrm{d} \mathbf{x} .
\end{aligned}
$$

Curiously, equation (4.19) provides a proof that the limit of $n / t_{n}$ exists and is independent of $\mathbf{x}_{0}$. Indeed, set $\Phi(\mathbf{x}) \equiv 1$ and observe that two other limits in equation (4.19) obviously exist. Thus, the limit of the sequence $\left(n / t_{n}\right)$ is the same as for the case $\mathbf{x}_{0}=0$, which is covered by Proposition 4.3 giving the limit $\mathscr{L} / \pi$.

\section{Applichtions}

With the tools developed in Section 4 we can prove Theorems 3.1 and 3.4 While the proofs follow the same set of basic ideas, the eigenvector statistics proof is slightly simpler, and thus we present it first.

5.1. Eigenvector statistics. In this section we will prove Theorem 3.4 We will do it by introducing a family of spectra, $\left\{\lambda_{\alpha, n}\right\}$ indexed by $\alpha \in[0,2 \pi)$. We remind the reader that $\lambda_{n}$ were defined as solutions of the equation $\operatorname{det}\left[\mathrm{e}^{\mathrm{i} \lambda \mathbf{L}} S_{0}-I\right]=0$. We extend this definition and denote by $\lambda_{\alpha, n}$ the solutions of the equation

$$
\operatorname{det}\left[\mathrm{e}^{-\mathrm{i} \alpha} \mathrm{e}^{\mathrm{i} \lambda \mathrm{L}} S_{0}-I\right]=0
$$

Thus, $\lambda_{\alpha, n} \mathbf{L}-\boldsymbol{\alpha} \in \Sigma$, where $\boldsymbol{\alpha}=\alpha(1, \ldots, 1)$ and $\Sigma$ is the surface defined by (4.1). In other words, $\lambda_{\alpha, n}$ are the intersection times of the flow $\phi_{t}(-\boldsymbol{\alpha})$ with $\Sigma$.

Similar to $\phi_{n}$, we can now define $\phi_{\alpha, n}$ to be the eigenvector of eigenvalue 1 of the matrix $\mathrm{e}^{\mathrm{i} \alpha} \mathrm{e}^{\mathrm{i} \lambda_{\alpha, n} \mathbf{L}} S_{0}$. Obviously, $\lambda_{n}=\lambda_{0, n}$ and $\phi_{n}=\phi_{0, n}$. We let $A_{\alpha, n}:=$ $\left\langle\phi_{\alpha, n}|A| \phi_{\alpha, n}\right\rangle$. 
Lemma 5.1. Let $G$ be a bounded continuous and possibly non-linear functional on $\mathbb{C}^{2 B}$. For $\mathbf{x} \in \Sigma$ define $\Phi(\mathbf{x}):=G\left(\phi_{\mathbf{x}}\right)$, where $\phi_{\mathbf{x}}$ satisfies

$$
\mathrm{e}^{\mathrm{ix}} S_{0} \phi_{\mathbf{x}}=\phi_{\mathbf{x}} \text {. }
$$

Then

$$
\lim _{N \rightarrow \infty} \frac{1}{N} \sum_{n=1}^{N} G\left(\phi_{\alpha, n}\right)=\frac{1}{(2 \pi)^{B} \epsilon} \int_{\mathbb{T}^{B}} \Phi_{\epsilon}(\mathbf{x}) \mathrm{d} \mathbf{x} .
$$

Proof. First we remark that $\Phi(\mathbf{x})$ is well-defined and continuous on $\Sigma$, except possibly at degenerate points of $\Sigma$.

By definition, $\phi_{\alpha, n}=\phi_{\mathbf{x}}$ with $\mathbf{x}=\lambda_{\alpha, n} \mathbf{L}-\boldsymbol{\alpha}$. Therefore,

$$
G\left(\phi_{\alpha, n}\right)=\Phi\left(\lambda_{\alpha, n} \mathbf{L}-\boldsymbol{\alpha}\right)=\Phi\left(\phi_{\lambda_{\alpha, n}}(-\boldsymbol{\alpha})\right) .
$$

Now we can apply Proposition 4.4 to conclude the proof.

The important consequence of Lemma 5.1 is that the moments of $A_{\alpha, n}$ are independent of $\alpha$.

Proof of Theorem 3.4. Let $\left\{\mathrm{e}^{\mathrm{i} \theta_{j}(\lambda)}\right\}_{j=1}^{2 B}$ be the eigenvalues of the matrix $\mathrm{e}^{\mathrm{i} \lambda \mathbf{L}} S_{0}$, with $\theta_{j}(\lambda)$ chosen to be real analytic. As a consequence of Lemma 4.1, $\theta_{j}$ has a smooth inverse.

Using a $2 \pi$-periodized Dirac delta function, $\delta_{2 \pi}$, we can trivially write

$$
A_{j}(\lambda)^{m}=\int_{0}^{2 \pi} \delta_{2 \pi}\left(\alpha-\theta_{j}(\lambda)\right) A_{j}(\lambda)^{m} \mathrm{~d} \alpha .
$$

Then

$$
\begin{aligned}
\int_{0}^{\Lambda} \sum_{j=1}^{2 B} A_{j}(\lambda)^{m} \mathrm{~d} \lambda & =\int_{0}^{2 \pi} \sum_{j=1}^{2 B} \int_{0}^{\Lambda} \delta_{2 \pi}\left(\alpha-\theta_{j}(\lambda)\right) A_{j}(\lambda)^{m} \mathrm{~d} \lambda \mathrm{d} \alpha \\
& =\int_{0}^{2 \pi} \sum_{j=1}^{2 B} \int_{\theta_{j}(0)}^{\theta_{j}(\Lambda)} \delta_{2 \pi}(\alpha-\xi) \frac{A_{j}\left(\theta_{j}^{-1}(\xi)\right)^{m}}{\theta_{j}^{\prime}\left(\theta_{j}^{-1}(\xi)\right)} \mathrm{d} \xi \mathrm{d} \alpha . \\
& =\int_{0}^{2 \pi} \sum_{0<\lambda_{\alpha, n} \leq \Lambda} \frac{A_{j_{\alpha, n}}\left(\lambda_{\alpha, n}\right)^{m}}{\theta_{j_{\alpha, n}}^{\prime}\left(\lambda_{\alpha, n}\right)} \mathrm{d} \alpha .
\end{aligned}
$$

We have chosen $j_{\alpha, n}$ so that $\theta_{j_{\alpha, n}}\left(\lambda_{\alpha, n}\right)=\alpha \bmod 2 \pi$. Therefore $\boldsymbol{\psi}_{j_{\alpha, n}}\left(\lambda_{\alpha, n}\right)$ is what we denoted by $\phi_{\alpha, n}$. Further, by Lemma 4.1, $\theta_{j}^{\prime}\left(\lambda_{\alpha, n}\right)=\left\langle\phi_{\alpha, n}|L| \phi_{\alpha, n}\right\rangle$. We set

$$
G(\phi)=\frac{\langle\phi|A| \phi\rangle^{m}}{\langle\phi|\mathbf{L}| \phi\rangle}, \quad \text { so that } \quad \frac{A_{j_{\alpha, n}}\left(\lambda_{\alpha, n}\right)^{m}}{\theta_{j_{\alpha, n}}^{\prime}\left(\lambda_{\alpha, n}\right)}=G\left(\phi_{\alpha, n}\right)
$$

and obtain

$$
\begin{aligned}
\int_{0}^{\Lambda} \sum_{j=1}^{2 B} A_{j}(\lambda)^{m} \mathrm{~d} \lambda & =\int_{0}^{2 \pi} \sum_{\lambda_{\alpha, n} \leq \Lambda} G\left(\phi_{\alpha, n}\right) \mathrm{d} \alpha \\
& =N(\Lambda) \int_{0}^{2 \pi} \frac{1}{N(\Lambda)} \sum_{\lambda_{\alpha, n} \leq \Lambda} G\left(\phi_{\alpha, n}\right) \mathrm{d} \alpha .
\end{aligned}
$$


Now we divide by $2 B \Lambda$ and take the limit $\Lambda \rightarrow \infty$. By Proposition $4.3 . N(\Lambda) / \Lambda \rightarrow$ $\mathscr{L} / \pi$ in this limit. By the dominated convergence theorem we can take the limit inside the $\alpha$-integral to get

$$
\begin{aligned}
\lim _{\Lambda \rightarrow \infty} \frac{1}{\Lambda} \int_{0}^{\Lambda} \frac{1}{2 B} \sum_{j=1}^{2 B} A_{j}(\lambda)^{m} \mathrm{~d} \lambda & =\frac{\mathscr{L}}{2 B \pi} \int_{0}^{2 \pi} \lim _{\Lambda \rightarrow \infty} \frac{1}{N(\Lambda)} \sum_{\lambda_{\alpha, n} \leq \Lambda} G\left(\phi_{\alpha, n}\right) \mathrm{d} \alpha \\
& =\frac{\mathscr{L}}{B} \lim _{\Lambda \rightarrow \infty} \frac{1}{N(\Lambda)} \sum_{n=1}^{N(\Lambda)} G\left(\phi_{0, n}\right) \\
& =\lim _{N \rightarrow \infty} \frac{1}{N} \sum_{n=1}^{N} \frac{A_{n}^{m}}{L\left(\lambda_{n}\right) / \bar{L}},
\end{aligned}
$$

where we used the fact that, by Lemma 5.1, the limit inside the integral is independent of $\alpha$.

To prove the second equality in (3.4) we apply Weyl's equidistribution theorem to the limit on the left in (5.5). This is justified since eigenvector components of $\mathrm{e}^{\mathrm{ix}} S_{0}$ vary continuously in $\mathbf{x}$ except possibly at the points where the eigenvalues of $\mathrm{e}^{\mathrm{ix}} S_{0}$ are non-simple. The set of such points is a $C$-analytic set and will have either measure 0 or full measure (see the discussion in Section [5.3). The latter situation is incompatible with condition (4.12).

5.2. Spectral statistics. We will use the previous results to prove that the spectral statistics of the matrix $\mathrm{e}^{\mathrm{ix}} S_{0}$ averaged over $\mathbb{T}^{B}$ converge to the empirical spectral statistics as the interval from which bond lengths are drawn shrinks in size.

5.2.1. A few more definitions. Let $d: \mathbb{T}^{B} \rightarrow \mathbb{R}_{+}$be the "time" of the next crossing of the surface $\Sigma$,

$$
d(\mathbf{x}):=\inf \left\{t>0: \phi_{t}(\mathbf{x}) \in \Sigma\right\} .
$$

For $\mathbf{x} \in \mathbb{T}^{B}$ let the eigenvalues of $\mathrm{e}^{\mathrm{i} \mathbf{x}} S_{0}$ be $\mathrm{e}^{\mathrm{i} \hat{\theta}_{1}(\mathbf{x})}, \ldots, \mathrm{e}^{\mathrm{i} \hat{\theta}_{2 v}(\mathbf{x})}$, ordered so that

$$
0<\hat{\theta}_{2 B}(\mathbf{x}) \leq \hat{\theta}_{2 B-1}(\mathbf{x}) \leq \cdots \leq \hat{\theta}_{2}(\mathbf{x}) \leq \hat{\theta}_{1}(\mathbf{x}) \leq 2 \pi .
$$

This ordering ensures that if $\mathbf{x} \in \Sigma$, then $\hat{\theta}_{1}(\mathbf{x})=2 \pi$. We use the hat in notation $\hat{\theta}_{j}$ to distinguish it from the eigenphases $\theta_{j}(\lambda)$ of the matrix $\mathrm{e}^{\mathrm{i} \lambda \mathbf{L}} S_{0}$ which can be chosen real analytic with respect to $\lambda$. Of course, the sets $\left\{\theta_{j}(\lambda)\right\}$ and $\left\{\hat{\theta}_{j}\left(\phi_{\lambda}(\mathbf{0})\right)\right\}$ coincide modulo $2 \pi$.

Define the eigenphase spacing functions by

$$
\begin{aligned}
\sigma_{1}(\mathbf{x}) & :=\hat{\theta}_{1}(\mathbf{x})-\hat{\theta}_{2}(\mathbf{x}) \\
& \vdots \\
\sigma_{2 B-1}(\mathbf{x}) & :=\hat{\theta}_{2 B-1}(\mathbf{x})-\hat{\theta}_{2 B}(\mathbf{x}) \\
\sigma_{2 B}(\mathbf{x}) & :=\hat{\theta}_{2 B}(\mathbf{x})+2 \pi-\hat{\theta}_{1}(\mathbf{x}) .
\end{aligned}
$$

These definitions are illustrated in Figure 3 .

We will use the shorthand $\sigma_{j}(\lambda)$ for $\sigma_{j}\left(\phi_{\lambda}(\mathbf{0})\right)$ and $d(\lambda)$ for $d\left(\phi_{\lambda}(\mathbf{0})\right)$. 


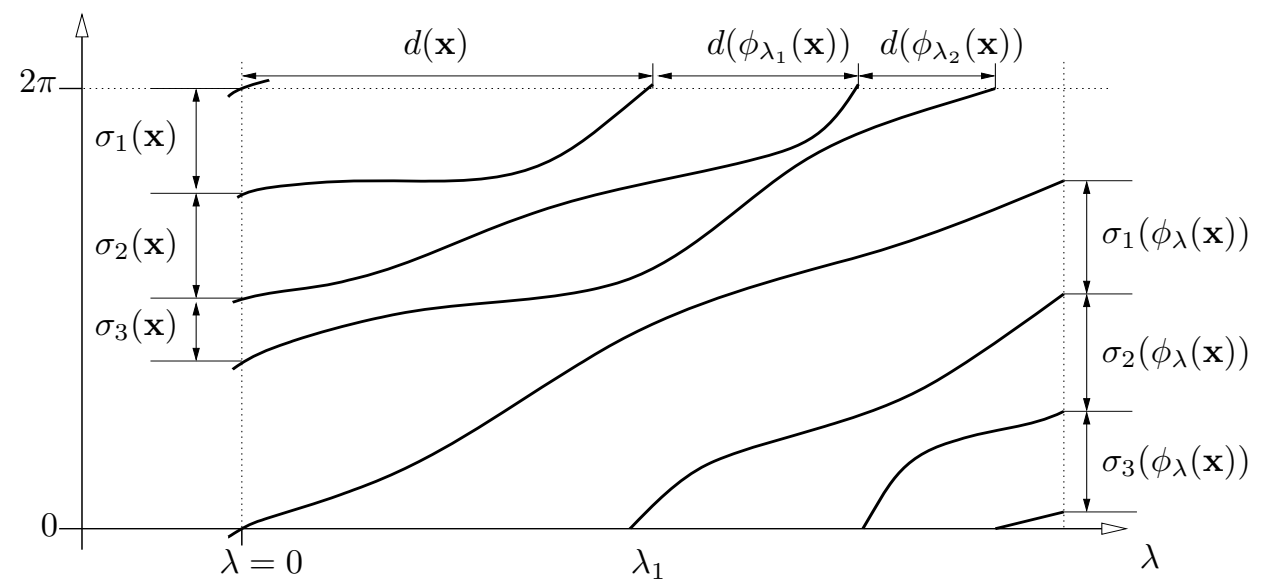

FIGURE 3. The eigenangles of $\mathrm{e}^{\mathrm{i} \phi_{\lambda}(\mathbf{x})} S_{0}$ and notation of Section 5.2.1.

\subsubsection{Equivalence of spacing distributions.}

Proof of Theorem 3.1. Let $h$ be a continuous test function. We want to compare

$$
\lim _{\Lambda \rightarrow \infty} \frac{1}{\Lambda} \int_{0}^{\Lambda} \frac{1}{2 B} \sum_{j=1}^{2 B} h\left(\sigma_{j}(\lambda)\right) \mathrm{d} \lambda \quad \text { and } \quad \lim _{N \rightarrow \infty} \frac{1}{N} \sum_{n=1}^{N} h\left(\bar{L} d\left(\lambda_{n}\right)\right) .
$$

Repeating the arguments of equations (5.3) and (5.4) we obtain

$$
\begin{aligned}
\int_{0}^{\Lambda} \sum_{j=1}^{2 B} h\left(\sigma_{j}(\lambda)\right) \mathrm{d} \lambda & =\int_{0}^{\Lambda} \int_{0}^{2 \pi} \sum_{r=1}^{2 B} \delta_{2 \pi}\left(\alpha-\theta_{r}(\lambda)\right) h\left(\sigma_{r}(\lambda)\right) \mathrm{d} \alpha \mathrm{d} \lambda \\
& =\int_{0}^{2 \pi} \sum_{n=1}^{\infty} \int_{0}^{\Lambda} \delta\left(\lambda-\lambda_{\alpha, n}\right) \frac{h\left(\sigma_{j_{\alpha, n}}(\lambda)\right)}{\theta_{j_{\alpha, n}}^{\prime}(\lambda)} \mathrm{d} \lambda \mathrm{d} \alpha \\
& =\int_{0}^{2 \pi} \sum_{\lambda_{\alpha, n} \leq \Lambda} \Phi^{\sigma}\left(\phi_{\lambda_{\alpha, n}}(-\boldsymbol{\alpha})\right) \mathrm{d} \alpha
\end{aligned}
$$

where, for $\mathbf{x} \in \Sigma$, we have defined

$$
\Phi^{\sigma}(\mathbf{x})=\frac{h\left(\sigma_{1}(\mathbf{x})\right)}{\left.\frac{\mathrm{d}}{\mathrm{d} t} \hat{\theta}_{1}\left(\phi_{t}(\mathbf{x})\right)\right|_{t=0}},
$$

where the derivative is taken from the left, since $\hat{\theta}_{1}$ is discontinuous on $\Sigma$. The function $\Phi^{\sigma}(\mathbf{x})$ is well defined if at $\mathbf{x}$ we have $\hat{\theta}_{2}<\hat{\theta}_{1}=2 \pi$, i.e. the eigenvalue 1 of $\mathrm{e}^{\mathrm{ix}} S_{0}$ is simple. Now we divide by $2 B \Lambda$, take the limit $\Lambda \rightarrow \infty$ and use Proposition 4.4 to get, analogously to (5.5),

$$
\begin{aligned}
\lim _{\Lambda \rightarrow \infty} \frac{1}{\Lambda} \int_{0}^{\Lambda} \frac{1}{2 B} \sum_{j=1}^{2 B} h\left(\sigma_{j}(\lambda)\right) \mathrm{d} \lambda & =\frac{\mathscr{L}}{2 B \pi} \int_{0}^{2 \pi} \lim _{\Lambda \rightarrow \infty} \frac{1}{N(\Lambda)} \sum_{\lambda_{\alpha, n} \leq \Lambda} \Phi^{\sigma}\left(\phi_{\lambda_{\alpha, n}}(\boldsymbol{\alpha})\right) \mathrm{d} \alpha \\
& =\frac{\bar{L}}{(2 \pi)^{B} \epsilon} \int_{\mathbb{T}^{B}} \Phi_{\epsilon}^{\sigma}(\mathbf{x}) \mathrm{d} \mathbf{x} .
\end{aligned}
$$


On the other hand, again by Proposition 4.4 we have

$$
\lim _{N \rightarrow \infty} \frac{1}{N} \sum_{n=1}^{N} h\left(\bar{L} d\left(\lambda_{n}\right)\right)=\frac{1}{(2 \pi)^{B} \epsilon} \int_{\mathbb{T}^{B}} \Phi_{\epsilon}^{d}(\mathbf{x}) \mathrm{d} \mathbf{x},
$$

where

$$
\Phi^{d}(\mathbf{x})=h(\bar{L} d(\mathbf{x})) .
$$

Now we use that $\bar{L} \Phi^{\sigma}(\mathbf{x})-\Phi^{d}(\mathbf{x}) \rightarrow 0$ as $\Delta L \rightarrow 0$. This is proved in Lemma 5.2 below, implying

$$
\frac{\bar{L}}{(2 \pi)^{B} \epsilon} \int_{\mathbb{T}^{B}} \Phi_{\epsilon}^{\sigma}(\mathbf{x}) \mathrm{d} \mathbf{x}-\frac{1}{(2 \pi)^{B} \epsilon} \int_{\mathbb{T}^{B}} \Phi_{\epsilon}^{d}(\mathbf{x}) \mathrm{d} \mathbf{x} \rightarrow 0 .
$$

Since $\Phi^{d}(\mathbf{x})$ is clearly continuous in $\mathbf{L}$, the individual limits exist too, concluding the proof.

Lemma 5.2. Let $h$ be continuous. Then, for $\mathbf{x} \in \Sigma$ we have

$$
\frac{\sigma_{1}(\mathbf{x})}{L_{\max }} \leq d(\mathbf{x}) \leq \frac{\sigma_{1}(\mathbf{x})}{L_{\min }},
$$

and, therefore,

$$
\bar{L} \Phi^{\sigma}(\mathbf{x})-\Phi^{d}(\mathbf{x}) \rightarrow 0
$$

uniformly on $\Sigma$ as $\Delta L=L_{\max }-L_{\min } \rightarrow 0$.

Proof. Estimate (5.11) follows from Lemma 4.1. Indeed, $d(\mathbf{x})$ is the time required by $\hat{\theta}_{1}\left(\phi_{t}(\mathbf{x})\right)$ to reach $2 \pi$ from its initial value of $2 \pi-\sigma_{1}+0$ at the time $t=+0$ (see Figure 3), and Lemma 4.1 provides an estimate on the derivative of $\hat{\theta}_{1}$.

We can now write (5.11) as

$$
\sigma_{1}(\mathbf{x})\left(\frac{\bar{L}}{L_{\max }}-1\right) \leq \bar{L} d(\mathbf{x})-\sigma_{1}(\mathbf{x}) \leq \sigma_{1}(\mathbf{x})\left(\frac{\bar{L}}{L_{\min }}-1\right) .
$$

Since both $d$ and $\sigma_{1}$ are bounded, we have $\bar{L} d(\mathbf{x})-\sigma_{1}(\mathbf{x}) \rightarrow 0$ uniformly in $\mathbf{x}$ as the spread of the lengths decreases. The function $h$ is continuous on a compact set; therefore it is uniformly continuous and $h\left(\sigma_{1}(\mathbf{x})\right)-h(\bar{L} d(\mathbf{x})) \rightarrow 0$ uniformly in $\mathbf{x}$. Finally, the denominator in the definition of $\Phi^{\sigma}$ (see equation (5.8)) is bounded by $L_{\min }$ and $L_{\max }$ by Lemma 4.1 and thus converges to $\bar{L}$. All of the above put together imply (5.12).

5.3. Discontinuities of $\Phi^{\sigma}$ and $\Phi^{d}$. When defining the function $\Phi$ in Section 5.1 and the functions $\Phi^{\sigma}$ and $\Phi^{d}$ in Section 5.2 , we implicitly assumed that they satisfy condition (4.12) on the set of their discontinuities. In this section we explain why we can expect it in general and how to rectify the situation when the definitions of function $\Phi$ produce too many discontinuities.

The surface $\Sigma$ is a $C$-analytic set; see [31, Definition 6 , Chapter V]. Thus we can apply [31, Proposition 18, Chapter V] to it, which splits $\Sigma$ into an analytic manifold of dimension $B-1$ and the remainder $\mathcal{U}$. The remainder has a smaller dimension and obviously satisfies condition (4.12).

Consider a connected piece of the $(B-1)$-dimensional manifold. If on this piece the zero of the defining equation

$$
\operatorname{det}\left[\mathrm{e}^{\mathrm{i} \phi_{t}\left(\mathbf{x}_{0}\right)} S_{0}-I\right]=0
$$


is simple, there is a unique eigenvector $\phi_{\mathbf{x}}$ (see Section 5.1), continuous with respect to $\mathbf{x}$ on the manifold. The function $\Phi$ is well defined.

The situation when on all $(B-1)$-dimensional manifolds the eigenvalues are simple is expected to be generic. However, a proof exists only for a special case of graphs with Kirchhoff matching conditions [13]. Proving the simplicity (in a generic sense) of the solutions to

$$
\operatorname{det}\left[\mathrm{e}^{\mathrm{ix}} S_{0}-I\right]=0
$$

for general $S_{0}$ thus remains an important open question. To complicate the picture, there are counter-examples with persistent eigenvalues of multiplicity two: These are provided by graphs with a looping bond separated from the rest of the graph. Moreover, there are indications that the first-order perturbation used in [13] will not produce the result for general $S_{0}$.

Fortunately, when the multiplicity is greater than one on a $(B-1)$-dimensional submanifold of $\Sigma$, we can still make the functions $\Phi$ continuous. First we note that the multiplicity must be constant on the whole manifold. For simplicity we assume the multiplicity is two. The eigenspace of the eigenvalue 1 of the matrix $\mathrm{e}^{\mathrm{i} \phi_{t}\left(\mathbf{x}_{0}\right)} S_{0}$ is continuous, and we can choose a continuous basis $\phi_{\mathbf{x}, 1}$ and $\phi_{\mathbf{x}, 2}$. The function $\Phi$ of Section 5.1 can then be defined as

$$
\Phi(\mathbf{x})=G\left(\phi_{\mathbf{x}, 1}\right)+G\left(\phi_{\mathbf{x}, 2}\right)
$$

on the problematic manifold.

The function $\Phi^{d}$ of Section 5.2 can be simply defined as $h(\bar{L} d(\mathbf{x}))+h(0)$. To define the function $\Phi^{\sigma}$ in a continuous and meaningful manner, we note that if $\hat{\theta}_{1}(\mathbf{x})=\hat{\theta}_{2}(\mathbf{x})=2 \pi$, then the phases also coincide before the flow hits $\Sigma$, i.e. for small negative $t, \hat{\theta}_{1}\left(\phi_{t}(\mathbf{x})\right)=\hat{\theta}_{2}\left(\phi_{t}(\mathbf{x})\right)$. Thus the derivative in the denominator of equation (5.8) does not depend on which $\hat{\theta}$ we take, and one can set

$$
\Phi^{\sigma}(\mathbf{x})=\frac{h\left(\sigma_{1}(\mathbf{x})\right)+h(0)}{\left.\frac{\mathrm{d}}{\mathrm{d} t} \hat{\theta}_{1}\left(\phi_{t}(\mathbf{x})\right)\right|_{t=0}} .
$$

\section{ACKNOWLEDGMENTS}

We are grateful for a number of interesting discussions with J. Bolte, D. Grieser, J. Harrison, J.P. Keating and P. Kuchment.

This work was supported by the National Sciences Foundation under research grant DMS-0604859.

\section{REFERENCES}

1. F. Barra and P. Gaspard, On the level spacing distribution in quantum graphs, J. Stat. Phys. 101 (2000), no. 1-2, 283-319. MR.1807548 (2001k:81073)

2. G. Berkolaiko, Form factor expansion for large graphs: A diagrammatic approach, in Quantum graphs and their applications, 35-49, Contemp. Math., 415, Amer. Math. Soc., Providence, RI, 2006. MR2277606 (2007k:81066)

3. G. Berkolaiko, R. Carlson, S. Fulling, and P. Kuchment (eds.), Quantum graphs and their applications, Contemporary Mathematics, no. 415, AMS, 2006 (Proceedings of Joint Summer Research Conference, Snowbird, Utah). MR2279143 (2007f:81004)

4. G. Berkolaiko and J. P. Keating, Two-point spectral correlations for star graphs, J. Phys. A 32 (1999), no. 45, 7827-7841. MR.1732273(2000k:81075)

5. G. Berkolaiko, J. P. Keating, and U. Smilansky, Quantum ergodicity for graphs related to interval maps, Commun. Math. Phys. 273 (2007), 137-159. MR2308752 (2008d:81080) 
6. G. Berkolaiko, J. P. Keating, and B. Winn, No quantum ergodicity for star graphs, Commun. Math. Phys. 250 (2004), 259-285. MR.2094517(2005k:81085)

7. G. Berkolaiko, H. Schanz, and R. S. Whitney, Leading off-diagonal correction to the form factor of large graphs, Phys. Rev. Lett. 88 (2002), no. 10, art. no. 104101.

8. O. Bohigas, M.-J. Giannoni, and C. Schmit, Characterization of chaotic quantum spectra and universality of level fluctuation laws, Phys. Rev. Lett. 52 (1984), no. 1, 1-4. MR730191 (85f:58034)

9. G. Casati, F. Valz-Griz, and I. Guarneri, On the connection between the quantization of nonintegrable systems and statistical theory of spectra, Lett. Nuovo Cimento 28 (1980), 279282. MR.580056 (81m:81029)

10. Y. Colin de Verdière, Ergodicité et fonctions propres du Laplacien, Commun. Math. Phys. 102 (1985), 497-502. In French. MR818831 (87d:58145)

11. P. Exner, J. P. Keating, P. Kuchment, T. Sunada, and A. Teplayev (eds.), Analysis on graphs and its applications, Proceedings of Symposia in Pure Mathematics, vol. 77, AMS, 2008 (Proceedings of Isaac Newton Institute programme). MR2459860 (2009i:00008)

12. P. Exner and P. Šeba, Free quantum motion on a branching graph, Rep. Math. Phys. 28 (1989), 7-26. MR 1109248(92e:81019)

13. L. Friedlander, Genericity of simple eigenvalues for a metric graph, Israel J. Math. 146 (2005), 149-156. MR2151598(2006d:34063)

14. S. A. Fulling, P. Kuchment, and J. H. Wilson, Index theorems for quantum graphs, J. Phys. A 40 (2007), 14165-14180. MR.2438118 (2009e:34075)

15. N. I. Gerasimenko and B. S. Pavlov, A scattering problem on noncompact graphs, Teoret. Mat. Fiz. 74 (1988), no. 3, 345-359. In Russian. MR953298 (90f:47010)

16. S. Gnutzmann and A. Altland, Spectral correlations of individual quantum graphs, Phys. Rev. E 72 (2005), no. 5, art. no. 056215. MR.2198317 (2006i:81075)

17. S. Gnutzmann and U. Smilansky, Quantum graphs: Applications to quantum chaos and universal spectral statistics, Advances in Physics 55 (2006), no. 5, 527-625.

18. D. Grieser, Monotone unitary families, Preprint ArXiv:0711.2869.

19. J. S. Griffith, A free-electron theory of conjugated molecules. I. Polycyclic hydrocarbons, Trans. Faraday Soc. 49 (1953), 345-351.

20. M. Harmer, Hermitian symplectic geometry and extension theory, J. Phys. A 33 (2000), no. 50, 9193-9203. MR.1804888 (2001m:58009)

21. V. Kostrykin and R. Schrader, Kirchhoff's rule for quantum wires, J. Phys. A 32 (1999), no. 4, 595-630. MR $1671833(99 \mathrm{~m}: 81280)$

22. V. Kostrykin, J. Potthoff, and R. Schrader, Heat kernels on metric graphs and a trace formula, Adventures in Mathematical Physics (F. Germinet and P. D. Hislop, eds.), Contemporary Mathematics, no. 447, AMS, 2007, pp. 175-198. MR.2423580

23. T. Kottos and U. Smilansky, Quantum chaos on graphs, Phys. Rev. Lett. 79 (1997), 47944797.

24. _ Periodic orbit theory and spectral statistics for quantum graphs, Ann. Phys. 274 (1999), 76-124. MR1694731 (2000f:81058)

25. P. Kuchment, Graph models for waves in thin structures, Waves Random Media 12 (2002), no. 4, R1-R24. MR1937279 (2003h:35215)

26. __ Quantum graphs. I. Some basic structures, Waves Random Media 14 (2004), no. 1, S107-S128, Special section on quantum graphs. MR2042548 (2005h:81148)

27. L. Kuipers and H. Niederreiter, Uniform Distribution of Sequences, Pure and Applied Mathematics, John Wiley \& Sons, New York, 1974. MR.0419394(54:7415)

28. P. Kurasov and M. Nowaczyk, Inverse spectral problem for quantum graphs, J. Phys. A 38 (2005), no. 22, 4901-4915, Corrigendum Ibid 39 (2006) p. 993. MR 2148632

29. G. Lumer, Espaces ramifiés, et diffusions sur les réseaux topologiques, C. R. Acad. Sci. Paris Sér. A-B 291 (1980), no. 12, A627-A630. MR606449 (82a:31011)

30. M. L. Mehta, Random matrices, Pure and Applied Mathematics, vol. 142, Elsevier, 2004. MR 2129906 (2006b:82001)

31. R. Narasimhan, Introduction to the theory of analytic spaces, Lecture Notes in Mathematics, No. 25, Springer-Verlag, Berlin, 1966. MR0217337 (36:428)

32. S. Nicaise, Spectre des réseaux topologiques finis, Bull. Sci. Math. (2) 111 (1987), no. 4, 401-413. MR921561 (89a:58114)

33. L. Pauling, The dimagnetic entropy of aromatic molecules, J. Chem. Phys 4 (1936), 673-677. 
34. O. M. Penkin and Yu. V. Pokornyı̆, On a boundary value problem on a graph, Differentsial'nye Uravneniya 24 (1988), no. 4, 701-703, 734-735. In Russian. MR940760 (89h:34016)

35. J.-P. Roth, Le spectre du laplacien sur un graphe, Théorie du potentiel (Orsay, 1983), Lecture Notes in Math., vol. 1096, Springer, Berlin, 1984. In French, pp. 521-539. MR890375 (88f:58151)

36. K. Ruedenberg and C. W. Scherr, Free-electron network model for conjugated systems. I. Theory, J. Chem. Physics 21 (1953), no. 9, 1565-1581.

37. H. Schanz and U. Smilansky, Spectral statistics for quantum graphs: Periodic orbits and combinatorics, Phil. Mag. B 80 (2000), 1999-2021 (Proceedings of the Australian summer school on quantum chaos and mesoscopics).

38. A. I. Šnirel'man, Ergodic properties of eigenfunctions, Usp. Math. Nauk. 29 (1974), 181-182. MR 0402834 (53:6648)

39. G. Tanner, Spectral statistics for unitary transfer matrices of binary graphs, J. Phys. A 33 (2000), no. 18, 3567-3585. MR.1766442 (2001b:82021)

40. U Unitary-stochastic matrix ensembles and spectral statistics, J. Phys. A 34 (2001), no. 41, 8485-8500. MR 1876609 (2002k:82040)

41. J. von Below, A characteristic equation associated to an eigenvalue problem on $c^{2}$-networks, Linear Algebra Appl. 71 (1985), 309-325. MR813056 (87i:94030)

42. H. Weyl, Über die Gleichverteilung von Zahlen mod. Eins, Math. Ann. 77 (1916), 313-352. In German. MR 1511862

43. S. Zelditch, Uniform distribution of the eigenfunctions on compact hyperbolic surfaces, Duke Math. J. 55 (1987), 919-941. MR.916129 (89d:58129)

Department of Mathematics, Texas A\&M University, College Station, Texas 778433368

E-mail address: gregory.berkolaiko@math.tamu.edu

Department of Mathematics, Texas A\&M University, College Station, Texas 778433368

Current address: School of Mathematics, Loughborough University, Loughborough, LE11 3TU, United Kingdom 